Maryam Sadeghi* Axel Boese, Ivan Maldonado, Joerg Sauerhering, Simon Schlosser, Heinrich Wehberg, Konrad Wehberg, and Michael Friebe

\title{
Feasibility test of Dynamic Cooling for detection of small tumors in IR thermographic breast imaging
}

https://doi.org/10.1515/cdbme-2019-0100

\begin{abstract}
Thermographic imaging is a known technology to detect temperature differences. For medical applications, the patterns of heat distribution are used for diagnosis. It is already tested to visualize blood supply, inflammatory processes, and superficial or more extensive tumors, e.g. in the breast tissue. This method was promoted for breast screening purposes and as a substitute for mammography for mid aged women, but the results were not convincing for younger women, where tissue density is higher, tumor growth is often connected to local temperature increase and radiation-based mammography is not an option. Infrared (IR) thermography can support tumor screening. The screening should allow the early detection of small lesions even in the depth. Therefor we evaluated the feasibility of dynamic cooling in combination with IR imaging in a phantom study. A temperature-controllable gel phantom including a heating plate, a depth-adjustable heat source mimicking a tumor, and three sensors for temperature monitoring was built up. A raspberry pi device serves as a control unit to create a stable temperature balance comparable to a human breast. For the experiments, the tumor was placed in various depth. After cooling, the thermal recovery phase of the phantom was imaged using an IR camera and a webcam. A pixel-wise analysis of the IR data detects a higher gradient of temperature change in the tumor region. The experiments demonstrated the feasibility of tumor detection based on dynamic cooling and IR imaging.
\end{abstract}

Keywords: Dynamic thermography, Dynamic cooling, Infrared imaging, Breast cancer.

\footnotetext{
*Corresponding author: Maryam Sadeghi, Institute of medical technology, INKA, Otto-von-Guericke University, Magdeburg, Germany, e-mail: maryam.sadeghi@ovgu.de

Axel Boese, Ivan Maldonado, Michael Friebe, Institute of medical technology, INKA, Otto-von-Guericke University, Magdeburg, Germany

Joerg Sauerhering, Institute of Fluid Dynamics and Thermodynamics, Otto-von-Guericke University, Magdeburg, Germany

Simon Schlosser, GBN Systems GmbH, Germany Heinrich Wehberg, Konrad Wehberg, Medizintechnik Wehberg $\mathrm{GmbH}$, Germany
}

\section{Introduction}

Breast cancer is a leading cause of death among women. Early detection of breast cancer is the key to improve the survival rate by $95 \%$ [1]. Among different available screening methods, mammogram remains the gold standard. However, it comes with high false-negative rates [2], the danger of ionizing radiation and is uncomfortable for the patients. In addition, it is not an accepted screening method, to detect breast cancer in young women [3], women with radiologically dense breasts or in women with breast implants [4]. As an alternative method, dynamic thermography has been investigated over the recent decades for detection of breast cancer as it eliminates the drawbacks mentioned above. But the diagnostic value of this technique is highly dependent on the experience of the medical doctor. There is no standardization of the imaging procedure so far. The interpretation of breast thermography is challenging due to the complexity of vascular patterns (false negatives and false positives). Some tumor types (cold tumors) can not be detected (false negatives) [5] using IR. Furthermore, in the conventional thermography diagnosis, the patient is imaged after undressing and again after waiting in a cold environment, which is uncomfortable for the patient and not repeatable. Moreover, this method, does not allow detection of small lesions situated in $2-3 \mathrm{~cm}$ depth. To overcome these issues, the development of a user-friendly standardized system for breast screening purposes is necessary. Research suggests that a high thermal gradient for a hot spot over a tumor is the most important factor to differentiate malignancy from a benign condition [6]. It is also reported that in order to better differentiate breast cancer from benign breast disease, thermography should be done after applying cold stress and during thermal recovery $[7,8]$. When a breast is exposed to cold stress, the thermal pattern changes. After the stress is removed, the pattern gradually recovers. IR imaging of this recovery phase allows measurement of the dynamics in temperature change. This can be used for detection of hot spots. In this study, this hypothesis is evaluated on a phantom. The phantom is mimicking the breast with a depth adjustable tumor to investigate the feasibility of infrared (IR) imaging after applying dynamic cooling. 

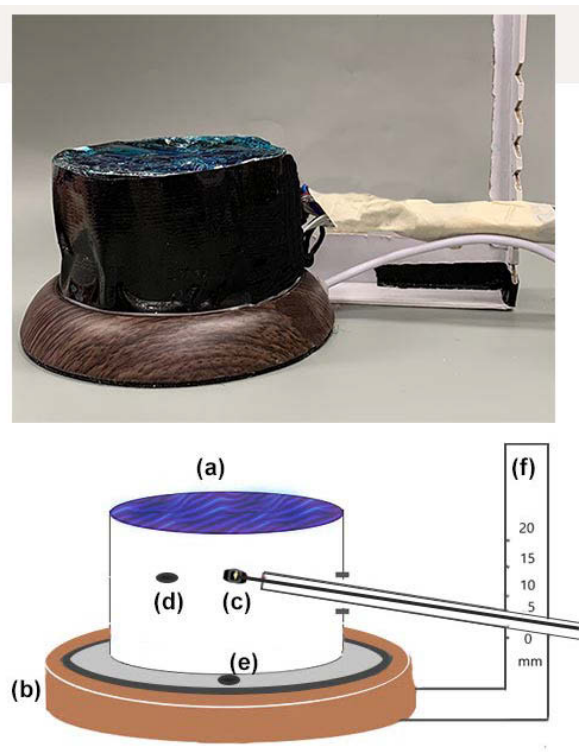

Fig. 1: The phantom comprises, of (a) cylinder containing temperature controllable gel, (b) heating plate, (c) a resistor together with a temperature sensor, (d) a temperature sensor for the surrounding gel, (e) a temperature sensor on the heating plate.

\section{Methods and Materials}

\subsection{Phantom}

To model the breast with a tumor, a phantom was built as shown in Figure 1. A cylinder container filled with temperature-controllable gel was placed on a heating plate. To model a tumor inside the phantom, a $1 \mathrm{k} \Omega$ resistor with the dimension of $2 \mathrm{~mm} \times 1 \mathrm{~mm}$ was used as heat source. The heat source was placed inside the gel with an attached temperature sensor, to monitor the temperature of the tumor. The depth of the resistor is adjustable in increments of $5 \mathrm{~mm}$. Two additional temperature sensors monitor the temperature of the gel and the heating plate.

\subsection{Image Acquisition}

An infrared camera FLIR A65 (FLIR Systems, Wilsonville, Oregon, United States) aligned with an optical webcam camera was placed above the phantom to acquire images simultaneously during and after applying the cold stress on the phantom. (see Figure 2)

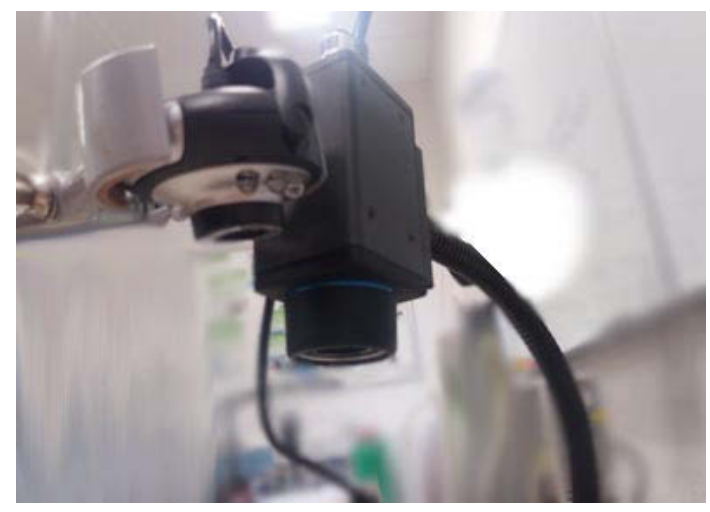

Fig. 2: The IR camera and webcam camera.

\subsection{Image Processing}

The sequence of images acquired from the IR camera was exported as csv files for each frame of the video. Each value of the array represented the temperature of the corresponding pixel in the image. The images were then analyzed based on the temperature change over time for different points. The temperature change of each pixel of the scene was plotted over time. To reduce the noisy pixels, averaging over grids of size $4 \mathrm{x} 4$ pixels was performed.

\subsection{Experimental Setup}

Figure 3 shows the equipment and the experimental setup for this study. The experiment was performed for tumor depth of 5 $\mathrm{mm}, 10 \mathrm{~mm}, 15 \mathrm{~mm}$ and $20 \mathrm{~mm}$. In each depth, cooling times of $3 \mathrm{~s}, 5 \mathrm{~s}, 7 \mathrm{~s}, 10 \mathrm{~s}$ were applied. In total 20 measurements were conducted. A box was placed around the phantom and the IR camera as a shield to eliminate the thermal reflections

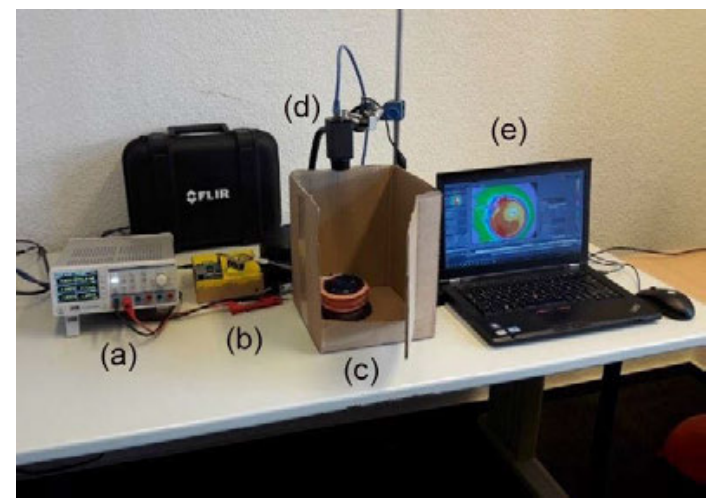

Fig. 3: The equipment and the experimental setup. (a) power supply, (b) Raspberry Pi 3 and control circuits, (c) phantom inside the box, (d) IR camera and webcam, (e) laptop 
from the surroundings. A Raspberry Pi3 device was used to acquire input from the three temperature sensors and adjust the temperature of the heating plate and the tumor object. The temperature of the tumor was set to $39^{\circ} \mathrm{C}$. The temperature of the gel surrounding the tumor was kept at $37^{\circ} \mathrm{C}$ by controlling the temperature of the heating plate. The IR camera was used to acquire images from the phantom. In this experiment, a glass container filled with water kept at $18{ }^{\circ} \mathrm{C}$ was used as the cooling apparatus. The water container was placed on the phantom for specific duration of time to apply cold stress. Room temperature during the experiments was $20-22{ }^{\circ} \mathrm{C}$.

\section{Results}

Figure 4 shows the temperature-time plot for 4 pixel bins in different areas of the image. The points were chosen in the tumor area, gel surrounding the tumor, the circumference of
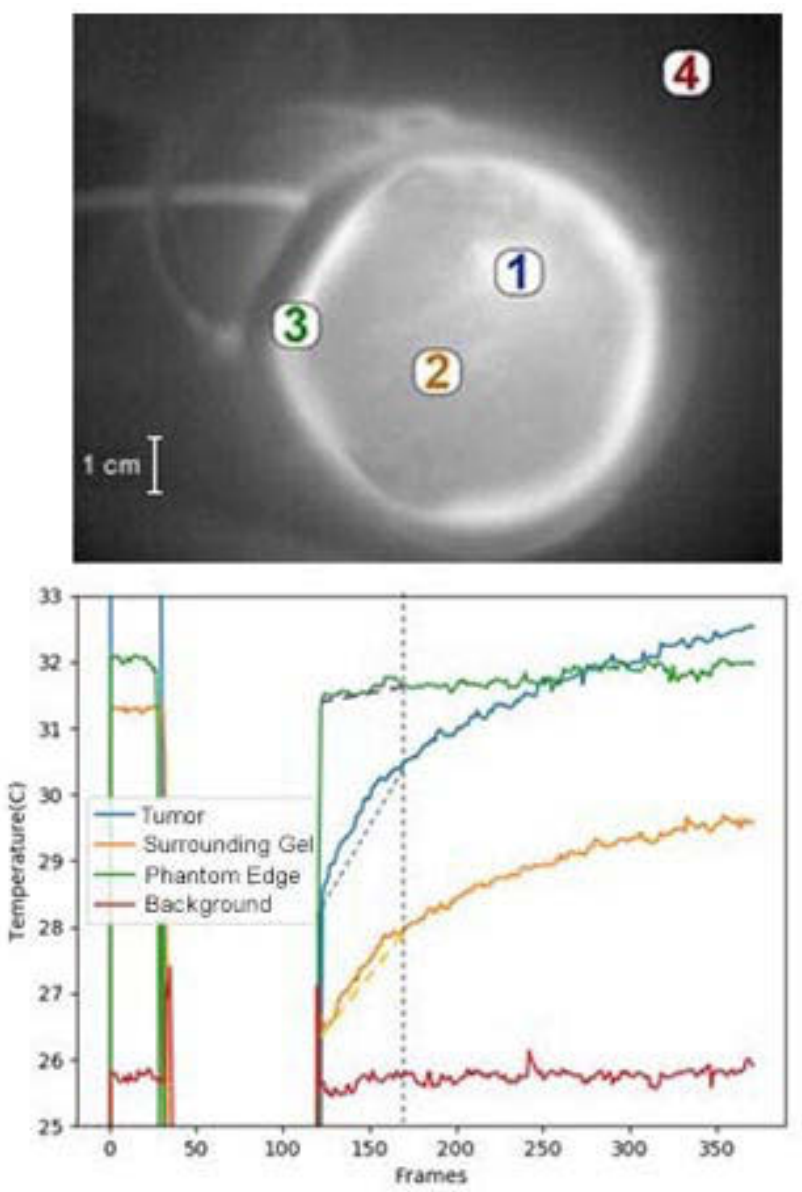

Fig. 4: The temperature-time plot for 4 different points in the scene. Tumor area, gel surrounding the tumor, the circumference of the phantom and background area.
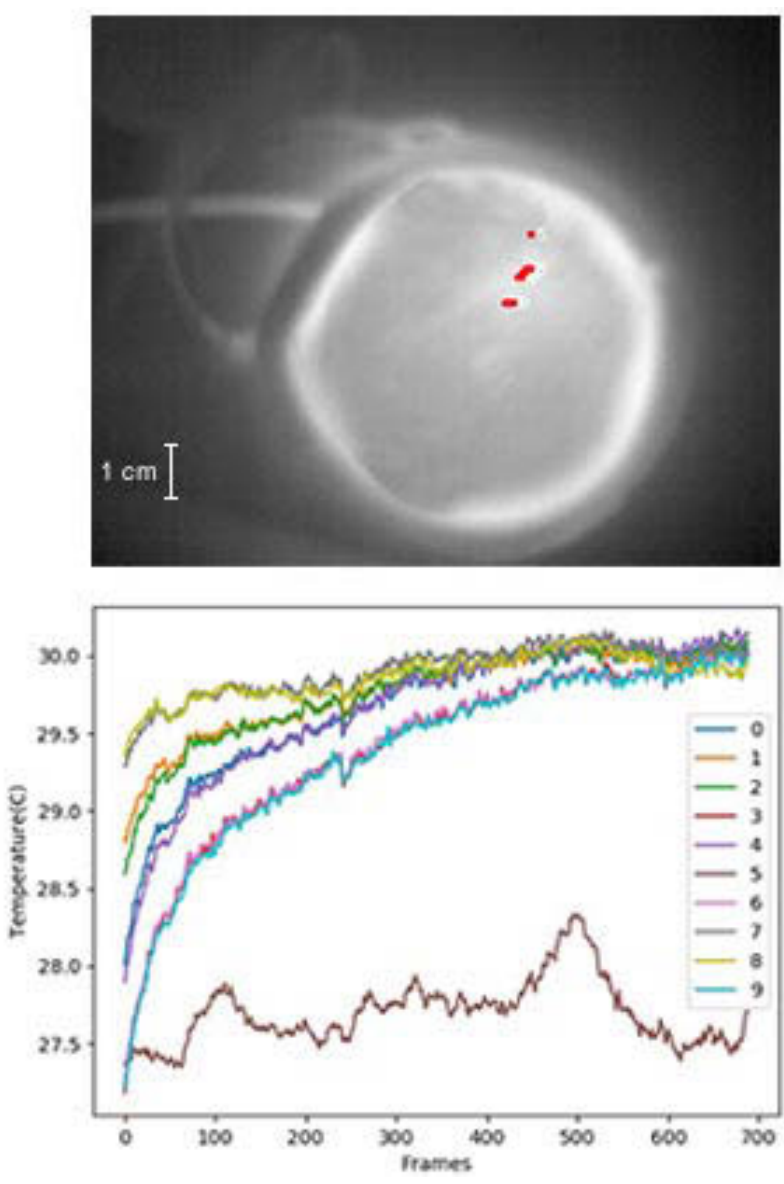

Fig. 5: Detection of 10 points with the highest thermal recovery gradient marked on the phantom image

the phantom and background area outside the phantom. It can be seen that the gradient of the thermal recovery is the highest at the tumor area.

The temperature behavior of $4 \times 4$ pixel areas of the image were plotted for all the pixel bins in the image and the 10 points with the biggest gradient were depicted in Figure 5. It can be seen that all points are correctly detected in the tumor area, except point number 5 , which is falsely marked due to its noisy nature.

Figure 6 shows the influence of tumor depth on the thermal recovery behavior. It was observed that increase in the tumor depth, results in the decrease of difference in thermal recovery gradient between the tumor and surrounding gel. 


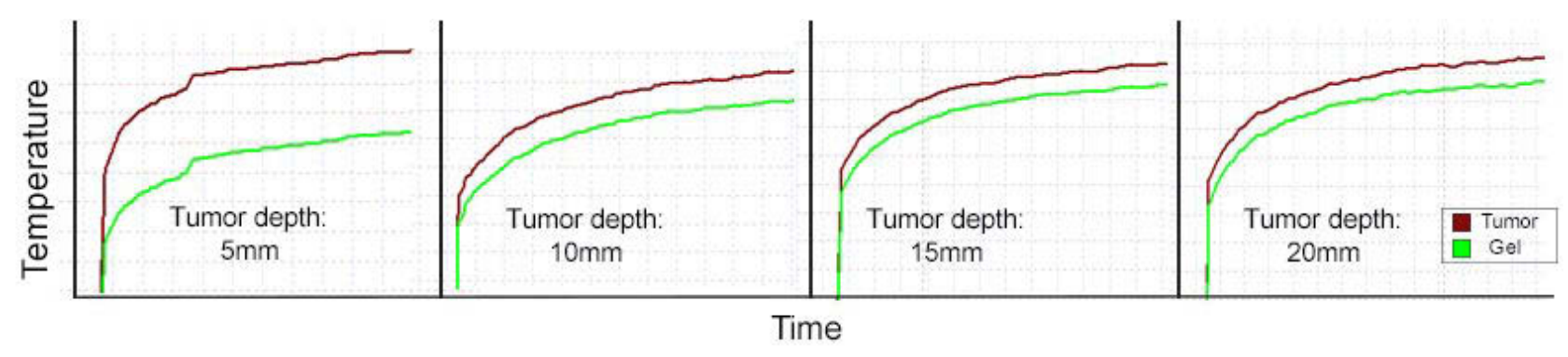

Fig. 6: The temperature-time plot for tumor and the surrounding gel, for tumor depth of $5 \mathrm{~mm}, 10 \mathrm{~mm}, 15 \mathrm{~mm}$ and $20 \mathrm{~mm}$

\section{Discussions and Conclusion References}

The experiments conducted in this study, demonstrated the feasibility of tumor detection based on dynamic cooling and IR imaging. Tumor depth influences the temperature spread on the surface over the tumor area, thus affecting the thermal recovery gradient between tumor and the surrounding gel. However tumor detection was still obtained with different tumor depths. Cooling time also influences thermal recovery time and gradient. It was observed that cooling time of 3 seconds was sufficient to achieve cold stress on the phantom. Reflections and sunlight can lead to irritations of the signal, this is a problem of the reflective surface of the phantom and does not occur on the human skin. Automatic tumor detection worked after pixel binning and gradient ranking.

\section{Author Statement}

Research funding: This research was financially supported by the Federal Ministry of Education and Research (BMBF) in the context of the 'INKA' project (Grand Number 03IPT7100X) and ZIM Früherkennung (ZF4206103AW6). ). We thank bgk infrarotservice GmbH for providing an IR camera for the experiments. Conflict of interest: Authors state no conflict of interest. Informed consent: Informed consent has been obtained from all individuals included in this study. Ethical approval: The research related to human use complies with all the relevant national regulations, institutional policies and was performed in accordance with the tenets of the Helsinki Declaration, and has been approved by the authors' institutional review board or equivalent committee.
[1] Tan, Tuan Zea, et al. "A novel cognitive interpretation of breast cancer thermography with complementary learning fuzzy neural memory structure." Expert Systems with Applications 33.3 (2007): 652-666.

[2] Elmore, Joann G., et al. "Variability in radiologists' interpretations of mammograms." New England Journal of Medicine 331.22 (1994): 1493-1499.

[3] Gohagan, John K., et al. "Individual and combined effectiveness of palpation, thermography, and mammography in breast cancer screening." Preventive medicine 9.6 (1980): 713-721.

[4] Foster, Kenneth R. "Thermographic detection of breast cancer." IEEE Engineering in medicine and biology Magazine 17.6 (1998): 10-14.

[5] Ohashi, Yasuhiko, and Isao Uchida. "Applying dynamic thermography in the diagnosis of breast cancer." IEEE Engineering in Medicine and Biology Magazine 19.3 (2000): 42-51.

[6] K Atsumi, "The diagnosis of breast cancer by thermography", Medical Thermography, pp. 215-252, 1973.

[7] K Atsumi, "Thermal recovery", Medical Thermography, pp. 151-165, 1973.

[8] J Steketee, MJ Van der Hoeck, "Thermal recovery of the skin after cooling", Phy Med Biol, vol. 24, pp. 583-587, 1979. 